\title{
Determination of rivaroxaban by different factor Xa specific chromogenic substrate assays: reduction of interassay variability
}

\author{
Job Harenberg • Roland Krämer • Christina Giese • \\ Svetlana Marx $\cdot$ Christel Weiss $\cdot$ Martin Wehling
}

Published online: 3 August 2011

(c) The Author(s) 2011. This article is published with open access at Springerlink.com

\begin{abstract}
Rivaroxaban and other oral direct factor Xa inhibitors (ODiXa) are currently developed for prophylaxis and treatment of thromboembolic diseases using fixed doses. Although routine monitoring is not required, assessing the intensity of anticoagulation may be useful under certain clinical conditions. ODiXa prolong coagulation times of several clotting assays and, thus, their concentration may be determined in factor Xa specific chromogenic substrate assays. So far, no standardized and validated assay is commercially available. Here, five methods (A through E) are studied and optimized to reduce interassay variability. Human pooled plasma was spiked by a serial dilution of rivaroxaban $(25-900 \mathrm{ng} / \mathrm{ml})$. The release of para-nitroaniline from the chromogenic substrates was measured by the optical density (OD) at $405 \mathrm{~nm}$. Method B was identified to yield the lowest sum of deviations from the mean value of the OD concentration curve calculated from all assays. Spline functions were
\end{abstract}

J. Harenberg $\cdot$ C. Giese $\cdot$ S. Marx $\cdot$ M. Wehling

Department of Clinical Pharmacology, University Heidelberg,

Maybachstrasse 14, 68169 Mannheim, Germany

\section{J. Harenberg $(\square)$}

Institute of Experimental and Clinical Pharmacology and

Toxicology, Clinical Pharmacology Mannheim, Medical Faculty

Mannheim, University of Heidelberg, Maybachstr 14, 68169

Mannheim, Germany

e-mail: Job.Harenberg@medma.uni-heidelberg.de

R. Krämer

Inorganic Chemistry Institute, University Heidelberg,

Im Neuenheimer Feld 270, 69120 Heidelberg, Germany

\section{S. Marx · C. Weiss}

Medical Statistics and Biomathematics, Faculty of Medicine

Mannheim, University Heidelberg, Maybachstrasse 14, 68169

Mannheim, Germany developed for OD versus concentration curves for all methods. The calculated OD versus concentration curves overlapped for all methods. The coefficient of variation for all assays and concentrations of rivaroxaban decreased from $25.3 \pm 11.4 \%$ using the original data to $3.8 \pm 2.2 \%$ using the calculated data $(P<0.0001)$. The robustness of the chromogenic assay (method B) remains to be corroborated in interlaboratory comparisons.

Keywords Rivaroxaban - Oral direct factor Xa inhibitors . Chromogenic assay $\cdot$ Factor Xa $\cdot$ Anticoagulants

\section{Introduction}

Oral direct factor Xa inhibitors (ODiXa) are currently developed for prevention and treatment of thromboembolic diseases with the aim to overcome the limitations of the conventional anticoagulants unfractionated and lowmolecular-weight heparins (LMWHs), fondaparinux, and vitamin-K antagonists [1]. One of the relevant advantages of ODiXa is the alleged dispensability of laboratory monitoring for dose adjustments [2, 3]. However, determination of the anticoagulant effect may be desirable in certain clinical situations such as acute bleeding, urgent surgery, analysis of compliance, suspected overdose, substantial decrease of renal or hepatic function, in elderly or children populations [4].

Attempts to assess anticoagulation intensity by ODiXa showed that inhibition of factor Xa activity, prolongation of prothrombin time (PT) and activated partial thromboplastin time (aPTT) correlate with plasma levels of the ODiXa (rivaroxaban in this case) in healthy subjects and those undergoing major orthopaedic surgery [5-8]. The prolongation of PT values by rivaroxaban depends of the 
thromboplastin reagents used [9]. Up to now, all chromogenic assays use heparin for standardization and data using rivaroxaban are sparse. A preliminary standardization of the reagents has been described using rivaroxaban [10]. Several factor Xa amidolytic chromogenic substrate assays have been tested to specifically determine the concentration of rivaroxaban; however, results of those methods show a considerable interassay variability of up to $40 \%$ [11]. The present paper aims at reducing the interassay variability between various chromogenic substrate assays on human plasma samples spiked with rivaroxaban.

\section{Materials and methods}

Rivaroxaban was isolated from commercially available Xarelto ${ }^{\circledR}$ (Bayer Healthcare Care, Wuppertal, Germany) by extracting an aqueous suspension of the tablets with dichloromethane at $25^{\circ} \mathrm{C}$. The compound was recrystallized from dichloromethane and vacuum-dried. The purity and identity were checked by $1 \mathrm{H}$ NMR spectroscopy, exact molecular mass by high-resolution electrospray mass spectrometry (Micromass Q-TOF ultima) and elemental analysis. Pooled plasma was spiked with increasing concentrations of rivaroxaban $(25-900 \mathrm{ng} / \mathrm{ml})$ dissolved in dimethyl sulfoxide (DMSO).

Blood was drawn from 20 healthy volunteers into $105 \mathrm{mmol} / 1$ sodium citrate (Sarstedt, Nuermbrecht, Germany), centrifuged at $3,000 \times g$ for $15 \mathrm{~min}$ at $4^{\circ}$ to obtain platelet poor plasma (PPP). Pooled plasma was derived from mixing PPP of 20 healthy persons. Plasma samples were aliquoted, transferred into plastic tubes, shock frozen and stored at $-70^{\circ}$ until analysed. Plasma samples were thawed only once at $37^{\circ}$, rivaroxaban was added at various concentrations, and analysed in the assays within $2 \mathrm{~h}$. Donors gave informed consent prior to blood sampling. Volunteers gave written informed consent.

\section{Chromogenic anti-Xa assays}

The test principle is based on the inhibitory action of rivaroxaban on coagulation factor $\mathrm{Xa}$ which specifically cleaves para-nitroaniline ( $p$-NA) linked to a chromogenic peptide. Increasing rivaroxaban concentrations dosedependently inhibit the activity of factor Xa on the chromogenic peptide and thereby the release of $p$-NA. The concentration of rivaroxaban is plotted against the optical density (OD) of released $p$-NA.

\section{Reagents}

The following factor Xa specific chromogenic substrates were used: Coamatic Heparin assay (method A, S-2732 chromogenic substrate, Suc-isoleucine-glutamyl(gammaPip)-glycine-arginine- $p N$-nitroaniline, Haemochrom Diagnostica GmbH, Essen, Germany), STA Rotachrom heparin (method B, chromogenic substrate CBS 52.44, MAPAglycyl-argininyl-p-nitroaniline hydrochloride, Diagnostica Stago, distributed by Roche-Diagnostika, Mannheim, Germany), S2222 chromogenic substrate assay (method C, $N$-benzoyl-L-isoleucyl-L-glutamylglycyl-L-arginine- $p$-nitroaniline hydrochloride and its methyl ester, Instrumentation Laboratory GmbH, Kirchheim, Germany), STA-heparin Liquid (method D, chromogenic substrate CBS-02.44, MAPA-glycine-arginyl-p-nitroanilide, Asnières sur Seine, France), and Technochrom anti-Xa (method E, chromogenic substrate succinyl-isoleucine-glutamyl-glycyl-arginine-p-nitroaniline, Technoclone, Vienna, Austria).

\section{Assay methodologies}

All reagents were dissolved in the solvent provided by and according to the description of the manufacturers. All assays were run on microtiter plates and not on the instruments proposed by the manufacturers. This was decided to eliminate the variability of the experiments caused by differences of the instructions by the manufacturers and coagulation analysers. Some manufacturers did not have instructions for the determination of rivaroxaban in the chromogenic assays. Preliminary experiments revealed that the maximal OD at $405 \mathrm{~nm}$ in the absence of rivaroxaban differed substantially between the assays using the incubation procedures described below. Therefore, the amounts of the chromogenic substrate and of factor Xa were adjusted for every method to about $1.000 \mathrm{OD}$ at $405 \mathrm{~nm}$ in the absence of rivaroxaban. The molar ratios of the substrate and factor $\mathrm{Xa}$ were not changed for the individual assays. $25 \mu \mathrm{l}$ human plasma containing rivaroxaban at various concentrations were diluted 1:5 with $25 \mu \mathrm{l}$ normal pooled plasma followed, $25 \mu \mathrm{l}$ factor $\mathrm{Xa}$ and incubated at $37^{\circ} \mathrm{C}$ for $5 \mathrm{~min} .50 \mu \mathrm{l}$ of synthetic chromogenic substrates were added and the samples incubated for $20 \mathrm{~min}$. Samples were supplemented with $25 \mu \mathrm{l}$ antithrombin (stock solution 1 unit per $\mathrm{ml}$ ) for the analysis with the technochrom anti-Xa assay before addition of factor $\mathrm{Xa}$, as recommended by the manufacturer. The enzymatic activity of factor Xa was stopped by adding $50 \mu \mathrm{l} 50 \%$ acetic acid. OD was recorded at $405 \mathrm{~nm}$ and converted to rivaroxaban $\mathrm{ng} / \mathrm{ml}$ plasma. Pooled plasma samples were spiked with $25-900 \mathrm{ng} / \mathrm{ml}$ rivaroxaban. Blank plasma was obtained by adding acetic acid prior to the chromogenic substrate to each plasma sample. No dilutions of samples containing high concentrations of rivaroxaban were performed in these experiments. The OD value of the plasma sample was subtracted from the OD of the test sample. The assays were performed on microtiter plates in duplicates 
and the absorbance of $p$-NA was read at a wavelength of $405 \mathrm{~nm}$ using the microtiter plate reader MR 7000 (software version 3.2, Dynatech laboratories, Denkendorf, Germany).

\section{Statistical analysis}

Summary statistics were computed for all OD values of all concentrations of rivaroxaban and all methods. The chromogenic assay with the lowest sum of the absolute differences from the mean value of the OD concentration curve calculated from all assays was defined as the reference method (B). Spline functions were determined for methods A, C, D, and E compared to method B. The $t$-test for two paired samples was used to compare the differences resulting from the data before and after introduction of the standard method. The level of significance was set at $P=0.01$. Statistical analyses were carried out using SAS/ STAT $^{\circledR}$ release 9.2 software (SAS Institute, Inc., Cary, NC, USA).

\section{Results}

Recrystallized rivaroxaban is a colorless solid powder of 99\% purity according to $1 \mathrm{H}$ NMR spectroscopy. Highresolution electrospray mass spectrometry (Micromass Q-TOF ultima) showed the identity of the compound. The exact molecular mass was calculated for $[\mathrm{M}+\mathrm{H}]+$ with 436.0740 (Ref. [12]: 436.0726), corresponding to $\mathrm{C}_{19} \mathrm{H}_{18} \mathrm{ClN}_{3} \mathrm{O}_{5} \mathrm{~S}$ with a molecular mass of 436.0734 . Elemental analysis determined the following $\mathrm{C} / \mathrm{H} / \mathrm{N}$ contents (in \%, calculated values in parenthesis): carbon 51.97 (52.36), hydrogen 4.23, nitrogen 9.54 (9.64). 1H NMR chemical shifts (200 MHz, Bruker Avance) of extracted rivaroxaban in $d_{6}$-DMSO were in agreement with literature data [12] (200 MHz, Bruker Avance).

Rivaroxaban was weighed using a calibrated analytical balance. $3.0 \mathrm{mg}$ Rivaroxaban were dissolved in $1 \mathrm{ml}$ dimethylsulfoxide (DMSO) and served as a stock solution. DMSO did not influence the chromogenic factor Xa assays up to $10 \mu \mathrm{l}$ per ml plasma (data not shown).

Rivaroxaban dose-dependently inhibited factor Xa in all chromogenic assays, i.e. in the presence of all chromogenic substrates, over the range of $25-900 \mathrm{ng} / \mathrm{ml}$ plasma.

The original OD concentration curves are depicted in Fig. 1. The coefficients of variation $(\mathrm{CV}$, mean values and $\mathrm{SD}$ ) of the methods using all ODs at all concentrations of rivaroxaban were: method A $3.36+2.19 \%$, method B $2.02 \pm 1.51 \%$, method C $5.03 \pm 4.79 \%$, method D $2.81 \pm$ $2.66 \%$, and method E $5.43 \pm 4.64 \%$, respectively. The CV value as well as the SD value was lowest for method $\mathrm{B}$.

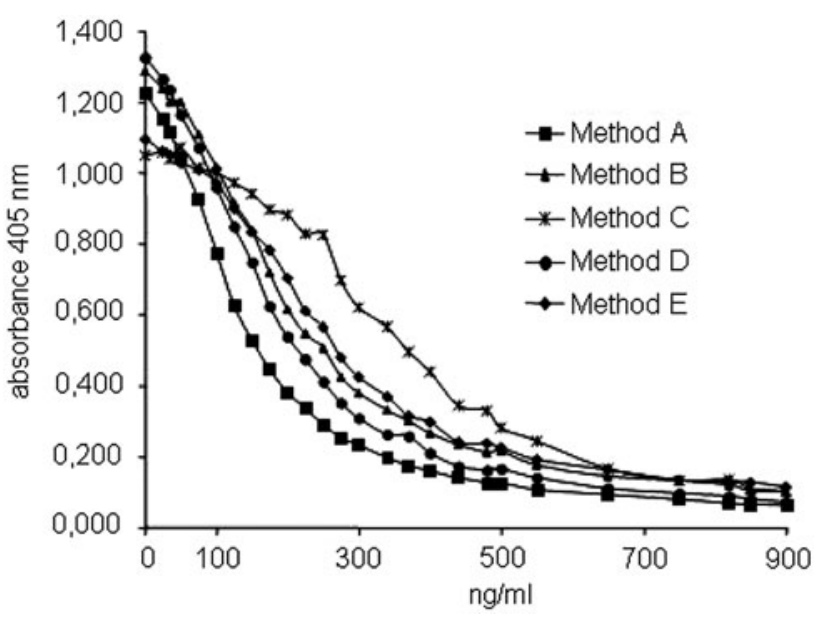

Fig. 1 OD concentration curves for five factor Xa specific chromogenic substrate methods as detailed in the method section

Calculating the difference of the individual methods versus the mean value of all OD concentration curves, method B turned out to expose the lowest sum of differences. Spline functions were calculated for methods A, C, $\mathrm{D}$, and $\mathrm{E}$ to minimize the difference against method $\mathrm{B}$ as reference (Table 1). Correction of the original OD concentration curves by these functions resulted in almost identical OD concentration curves for all methods (Fig. 2). The differences of the CVs (mean and SD) of the methods using the original data (Fig. 1, CV $25.3 \pm 11.4 \%$ ) and the calculated data (Fig. 2, CV $3.8 \pm 2.2 \%$ ) were significant $(P<0.001)$.

The OD curves for method B versus the other methods are shown in Fig. 3. Mathematical correction of the methods A, C, D, and E eliminates the differences seen in Fig. 3 (Fig. 4). The differences of the CVs (mean and SD) of the data between Fig. 3 (CV $28.76 \pm 13.53 \%)$ and Fig. 4 (CV $4.16 \pm 2.47 \%)$ were significant $(P<0.001)$.

Table 1 Mathematical functions for methods A, C, D, and E to reduce the differences compared to method $\mathrm{B}$

\begin{tabular}{|c|c|c|c|}
\hline Assay & Method & $\begin{array}{l}\text { Equations } \\
\text { versus } \\
\text { method B }\end{array}$ & $r^{2}$ \\
\hline Coamatic & A & $\begin{aligned} y & =0.2695 x^{3}-1.2234 x^{2} \\
& +2.1591 x-0.0379\end{aligned}$ & 0.9988 \\
\hline S2222 FXa & $\mathrm{C}$ & $\begin{aligned} \mathrm{y} & =3.239 \mathrm{x}^{3}-4.0879 \mathrm{x}^{2} \\
& +1.9418 \mathrm{x}-0.0724\end{aligned}$ & 0.9919 \\
\hline $\begin{array}{l}\text { STA liquid } \\
\text { anti Xa }\end{array}$ & $\mathrm{D}$ & $\begin{aligned} \mathrm{y} & =-0.0583 \mathrm{x}^{3}-0.1207 \mathrm{x}^{2} \\
& +1.2159 \mathrm{x}+0.0147\end{aligned}$ & 0.9994 \\
\hline Technochrom & E & $\begin{array}{l}\mathrm{y}=1.0851 \mathrm{x}^{3}-1.2646 \mathrm{x}^{2} \\
\quad+1.3108 \mathrm{x}-0.0331\end{array}$ & 0.9986 \\
\hline
\end{tabular}

This method was considered as provisional standard since it exposed the lowest difference sum compared to the mean OD concentration curve averaged from of all methods employed here 


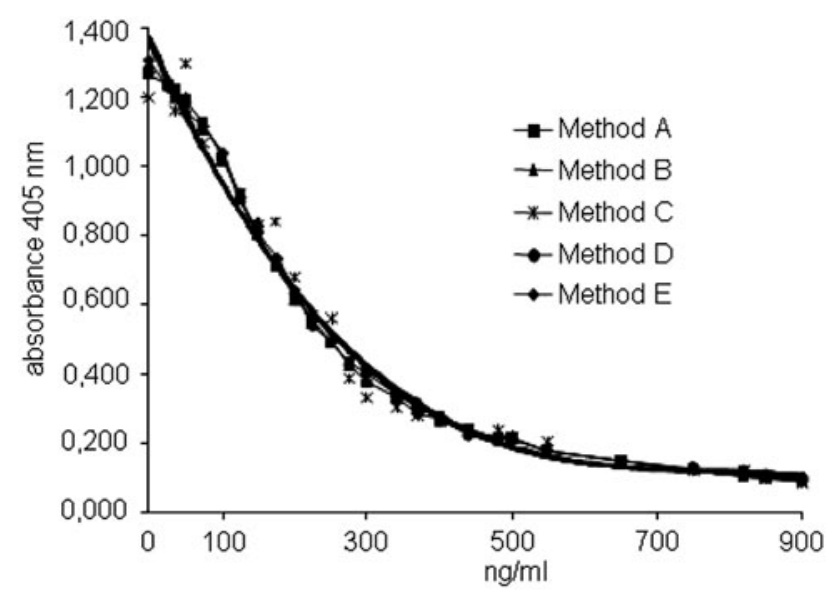

Fig. 2 Correction of the OD concentration curves of methods A, C, $\mathrm{D}$, and $\mathrm{E}$ by mathematical functions (see Table 1) to reduce the difference to method B (provisional standard method)

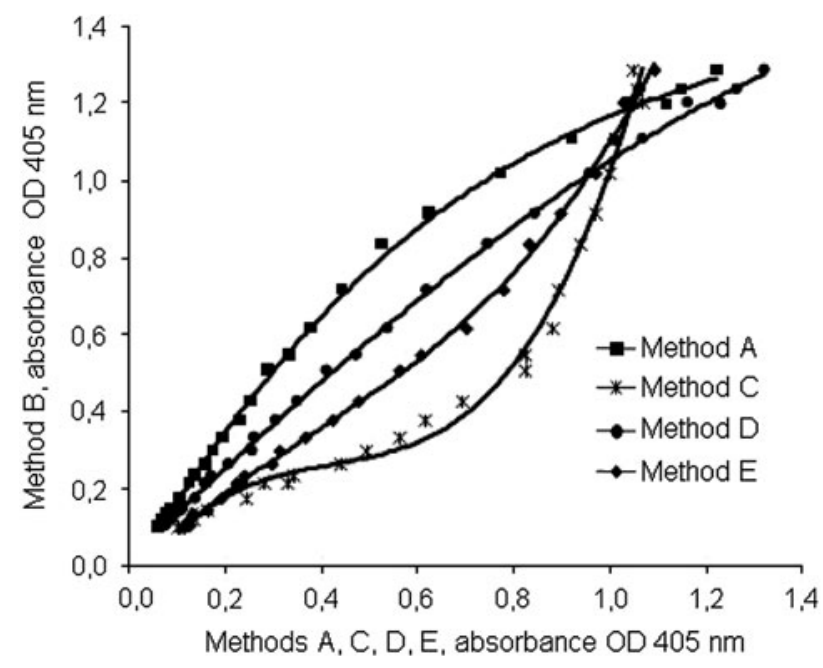

Fig. 3 OD of method B versus the OD of methods A, C, D, and E after normalization of the maximal OD for the same concentrations of rivaroxaban used in Figs. 1 and 2

\section{Discussion}

Factor Xa specific chromogenic assays, originally designed for indirect antithrombin dependent factor Xa inhibitors, and are variably influenced by rivaroxaban. The results of the present studies demonstrate that these differences can be eliminated by mathematical modelling. In addition, one assay (method B) was identified to possess the lowest variation from the mean OD concentration curve calculated from all five methods adopted in this study. A similar approach was described for thromboplastin reagents to determine the prothrombin time $[9,10]$. One of seven assays was identified to give the lowest variation when the other prothrombin time assays were compared to this alleged standard assay [11]. Another study used the

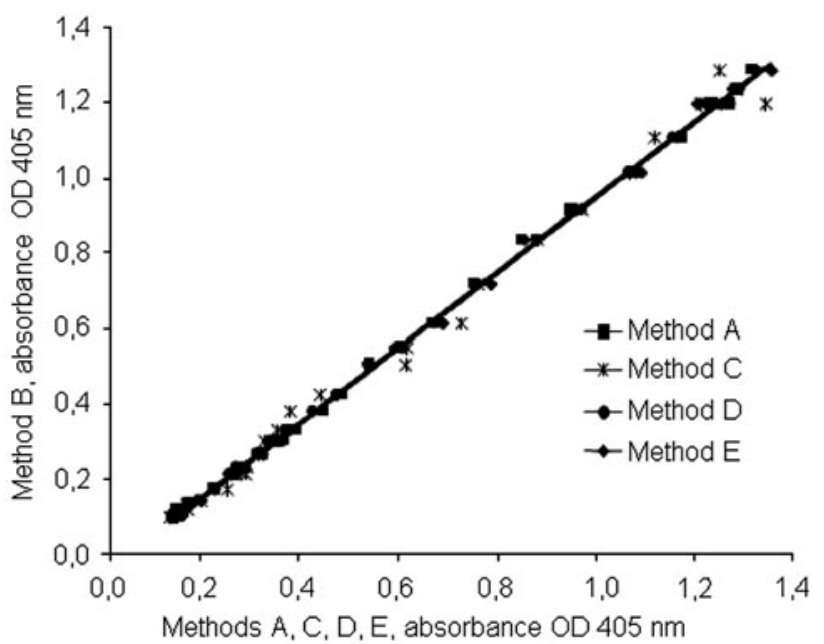

Fig. 4 Modification of the OD of method B versus the OD of methods A, C, D, and E by mathematical functions (see Table 1) to reduce the difference to method $\mathrm{B}$ for the same concentrations of rivaroxaban used in Figs. 1 and 2

WHO-thromboplastin reagent to compute the calculations for the commercially available thromboplastin reagents using plasma samples spiked with rivaroxaban [13]. Both approaches decreased the interassay variabilities. In the present investigations this was also shown for the comparison of different chromogenic assays. A validation of these results is currently ongoing in an interlaboratory control study [14].

Although routine monitoring is not expected to be needed for the new classes of small-molecule oral factor $\mathrm{Xa}$ inhibitors, a clinical interest in identifying reliable and readily available laboratory assays for measuring anticoagulant effects of the new class of oral factor Xa inhibitors is emerging. Clot-based methods such as prothrombin time assays and factor Xa chromogenic substrate assays appear to be the most reliable tools. Coagulation assays are inexpensive and easy to perform, but in contrast to chromogenic assays [15] they are affected by coagulation factor abnormalities [16, 17] or levels of plasma proteins [15].

The concentration ranges of the chromogenic assays used in this study should cover the range for low and high doses of rivaroxaban used to prevent or the treat acute thromboembolism. However, specific assay procedures have been described also for low and high concentration of rivaroxaban $[10,18]$. This may be preferable in a clinical routine laboratory. In our study, we adjusted the ratio of factor Xa and of the chromogenic substrate of every assay to obtain a similar maximal absorption by the release of the chromophore in the absence of rivaroxaban. This has to be performed for every assay by the manufacturer for routine application as well as the potential value of antithrombin added to the assay. The study presented here identifies an optimal chromogenic method to determine the 
concentration of rivaroxaban and can be adopted for additional chromogenic assays not included in our study.

Some limitations of the study have to be recognized. First, the different assays were designed to be run in ways which were as similar as possible; by this, it was not always possible to comply with the instructions of the manufacturers. This includes the fact that incubation times were the same for all methods, assays were run on the same instrument and the chromogenic factor $\mathrm{Xa}$ concentration modalities were modified to result in a similar OD in the absence of rivaroxaban. Only by doing this, the assays became comparable. Second, plasma samples were spiked with rivaroxaban and no samples were used from patients treated with rivaroxaban. These investigations are ongoing.

In summary, the present investigation demonstrates that differences between factor $\mathrm{Xa}$ chromogenic assays exist and that these differences can be reduced by mathematical modelling. Chromogenic substrate assays were made comparable for plasma samples spiked with rivaroxaban. Further studies are undertaken to corroborate the monocentric experience. Method validation needs to be extended to other chromogenic substrates, other coagulation analysers and samples from patients on rivaroxaban and its impact on factor Xa specific chromogenic substrate assays.

Conflict of interest The authors gratefully acknowledge the support of the reagents by the manufacturers. They do not have other conflicts of interests to declare.

Open Access This article is distributed under the terms of the Creative Commons Attribution Noncommercial License which permits any noncommercial use, distribution, and reproduction in any medium, provided the original author(s) and source are credited.

\section{References}

1. Weitz JI (2010) New oral anticoagulants in development. Thromb Haemost 103:62-70

2. Bounameaux H, Reber G (2010) New oral antithrombotics: a need for laboratory monitoring. J Thromb Haemost 8:627-630

3. Favaloro EJ, Lippi G (2011) Laboratory testing and/or monitoring of the new oral anticoagulants/antithrombotics: for and against? Clin Chem Lab Med 49:755-757

4. Mismetti P, Laporte S (2010) New oral antithrombotics: a need for laboratory monitoring. J Thromb Haemost 8:621-626

5. Mueck W, Becka M, Kubitza D et al (2007) Population model of the pharmacokinetics and pharmacodynamics of rivaroxaban-an oral, direct factor Xa inhibitor-in healthy subjects. Int $\mathrm{J}$ Clin Pharmacol Ther 45:335-344

6. Misselwitz F, Berkowitz SD, Perzborn E (2011) The discovery and development of rivaroxaban. Ann N Y Acad Sci 1222:64-75

7. Eriksson BI, Borris L, Dahl OE et al (2006) Oral, direct factor Xa inhibition with BAY 59-7939 for the prevention of venous thromboembolism after total hip replacement. J Thromb Haemost 4:121-128

8. Turpie AG, Fisher WD, Bauer KA et al (2005) BAY 59-7939: an oral, direct factor $\mathrm{Xa}$ inhibitor for the prevention of venous thromboembolism in patients after total knee replacement. A phase II dose-ranging study. J Thromb Haemost 3:2479-2486

9. Samama MM, Martinoli JL, LeFlem L, Guinet C et al (2010) Assessment of laboratory assays to measure rivaroxaban-an oral, direct factor Xa inhibitor. Thromb Haemost 103:815-825

10. Tripodi A, Chantarangkul V, Guinet C, Samama M (2011) The INR calibrated for rivaroxaban-(INRrivaroxaban) has the potential to normalize PT results for rivaroxaban-treated patients: results of an in vitro study. J Thromb Haemost 9:226-228

11. Barrett YC, Wang Z, Frost C, Shenker A (2010) Clinical laboratory measurement of direct factor Xa inhibitors: anti-Xa assay is preferable to prothrombin time assay. Thromb Haemost 104:1263-1271

12. Roehrig S, Straub A, Pohlmann J, Lampe T, Pernerstorfer J, Schlemmer KH, Reinemer P et al (2005) Discovery of the novel antithrombotic agent 5-chloro-N-(\{(5S)-2-oxo-3-[4-(3-oxomorpholin-4-yl)phenyl]-1, 3-oxazolidin-5-yl\}methyl)thiophene-2-carboxamide (BAY 59-7939): an oral, direct factor Xa inhibitor. J Med Chem 48:5900-5908

13. Harenberg J, Marx S, Krämer R, Giese C, Weiss C (2011) Determination of an international sensitivity index of thromboplastin reagents using a WHO thromboplastin as calibrator for plasma spiked with rivaroxaban. Blood Coagul Fibrinol. (In press)

14. Harenberg $\mathbf{J}$ (2011) Report of the working party on standardization of methods to determine direct factor Xa inhibitors http:// ssc2010.org/ssc2010/index.cfm/program/ssc-sessions/Monday24-may/Assessed 28 June, 2011

15. McGlasson DL, Kaczor DA, Krasuski RA et al (2005) Effects of pre-analytical variables on the anti-activated factor X chromogenic assay when monitoring unfractionated heparin and low molecular weight heparin anticoagulation. Blood Coagul Fibrinolysis $16: 173-176$

16. van Geest-Daalderop JH, Hutten BA, Péquériaux NC et al (2007) The influence on INRs and coagulation factors of the time span between blood sample collection and intake of phenprocoumon or acenocoumarol: consequences for the assessment of the dose. Thromb Haemost 4:738-746

17. Gerotziafas GT, Dupont C, Spyropoulos AC et al (2009) Differential inhibition of thrombin generation by vitamin $\mathrm{K}$ antagonists alone and associated with low molecular- eight heparin. Thromb Haemost 102:42-4818

18. Perzborn E, Harwardt M, Samama MM (2009) Assessment of factor Xa chromogenic assays for measuring the pharmacodynamics of rivaroxaban - an oral, direct factor Xa inhibitor, ISTH. J Thromb Haemost 7(Suppl 2) (Abstract PP-MO-185) 